The Indonesian J Dent Res, 2011, Volume 1, No. 3

Published online: http://the-indonesian-jdr.fkg.ugm.ac.id

\title{
The Newly Bone Formation with Carbonate Apatite-Chitosan Bone Substitute in the Rat Tibia
}

\author{
Anne Handrini Dewi ${ }^{1}$ \\ Andi Triawan ${ }^{2}$ \\ ${ }^{1}$ Departement of Biomedical Sciences, Faculty of Dentistry, \\ Universitas Gadjah Mada, Yogyakarta, Indonesia \\ ${ }^{2}$ Academic Hospital Universitas Gadjah Mada, Yogyakarta, Indonesia \\ E-mail: anne_ikgd@ugm.ac.id \\ Received April 11, 2011; Accepted May 11, 2011
}

\begin{abstract}
Large bone defect still represent a major problem in orthopedics. A tissue engineering approach has been proposed where osteogenic cells, bioceramic scaffolds and growth factors can play in a role to the bone repair. Bone consist a mineral phase such as carbonate apatite and an organic phase such as collagen. Synthetic carbonate apatite ceramics are considered as promising alloplastic materials for bone substitute. Chitin is the organic matrix of the hard parts of exoskeleton of insect, crustacean and present in a small amounts in mushrooms. It is an insoluble, similar to cellulose and composed of $\mathrm{N}$-acetylglucosamine unit. Partial deacetylation from chitin result in the formation of chitosan. Chitin's properties as a flexible and strong material make it favourable as surgical thread. It has novel properties such as biocompatibility, biodegradability, anti bacterial, wound healing activity, tissue regeneration and hemostatic activitities. The composit from carbonate apatite and chitosan may have a great impact on human health care system as bioresorbable bone substitute. The aim of the study was to evaluate the newly bone formation on the bone healing of defect tibia treated with carbonate apatite-chitosan bone substitute. Eighteen Sprague Dawley rats, male, 3 months, weighing 250-300g used in this study. Bilateral defect were created in each tibia rat. The defects were filled with carbonate-apatite chitosan bone substitute. The rats were sacrificed after respectively 1,2 and 3 weeks. The result of this study showed that carbonate apatite-chitosan significantly increased a number of osteoblast $(p<0.05)$. Carbonate apatite-chitosan group showed that matrix deposition faster than the other groups and have a good interface with the old bone. These data indicate that carbonate apatite-chitosan are potential candidate for bone substitute.
\end{abstract}

Key words: Carbonate apatite, chitosan, bone substitute.

\section{Introduction}

Bone is composite material made up of organic and inorganic components. By weight, bone is approximately $20 \%$ water. The mineral phase of bone (inorganic phase) comprised approximately 60 to $70 \%$ of the total dry bone weight while the remaining is comprised of organic materials (such as protein and collagen). The inorganic content of bone consists primary of calcium phosphate and calcium carbonate with small quantities of magnesium, fluoride and sodium ${ }^{1}$.

Bone repair or regeneration is a common and complex clinical problem in orthopedic and 
maxillofacial surgery. Every year, millions of people are suffering from bone defects arising from trauma, tumor or bone diseases ${ }^{2}$. Although the transplantation of autogenous vital bone is still the method of choice for the treatment of bony defects, alloplastic materials are often preferred because they are readily available and their use avoids a donor site defect. Several biomaterials have already been developed to fill and reconstruct a bone defect, such a natural coral, bovine porous demineralized bone, human demineralized bone matrix, bioactive glass ceramics, and calcium phosphate ceramics. Among these alternative materials, calcium phosphate ceramic (hydroxyapatite, $\alpha$-tricalcium phosphate, or biphasic calcium phosphate) occupy a prominent position $^{3,4,5,6}$.

For example, hydroxyapatite (HA) has been widely used as bone substitute because of its excellent biocompatibility and osteoconductivity. However, it has to be noticed that bone apatite contains carbonate and is therefore not pure hydroxyapatite ${ }^{7}$. Nonstoichiometric carbonated apatites (C-Ap) are the main mineral components of human and animal hard tissues (bones and teeth). They are increasingly used as biocompatible materials for medical purposes ${ }^{8}$. Due to its superior bioresorption, C-Ap is the preferred candidate material for bone reconstructive surgery ${ }^{7,9,10}$. The biodegradation of C-Ap occurs by osteoclasts ${ }^{3}$.

Chitosan has been researched for implant and wound healing applications. Chitosan, a natural biodegradable polymer, is a low acetyl substituted from of chitin named (1-4)-2-amino-2-deoxy-(Dglucose). Chitin has excellent biocompatibility for most tissue including skin and bone. Because of its unique properties such as biodegradability, non toxicity, anti-bacterial effect and biocompatibility, much attention has paid to chitosan-based biomedical materials ${ }^{11,12,13}$.

Since both materials have promising properties to be an ideal bone substitute material, a combination of carbonate apatite and chitosan prepared to be implanted in the rat tibia and will examine the bone regeneration after 1, 2 and 3 weeks.

\section{Materials and Methods}

\subsection{Materials}

Chitosan (90\% degree of deacetylation) was purchased from UPT, BBPTK, LIPI, Yogyakarta, Indonesia. Carbonate apatite was made by mixing 1.825 gram of calcium hydroxide with $100 \mathrm{ml}$ distilled water while $1.024 \mathrm{ml}$ of phosporic acid mixed with $100 \mathrm{ml}$ of distilled water. The calcium hydroxide solvent was placed in the stirring machine and stirred with the temperature set at $45^{\circ} \mathrm{C}$ for 2 hours meanwhile the acid phosporic mixture was titrated into the calcium hydroxide solvent ${ }^{9}$. The titrate mixture was tested for its $\mathrm{pH}$ where it was favored to be in the range of 6.5-7.5 which was neutral or slighty base. Next the mixture was placed into an oven with the temperature set at $60^{\circ} \mathrm{C}$ for 48 hours. Finally the dried substance was taken out and thumped and made into thin powder. The other material used in this study was petroleum white base purchased from Betraco Chemical.

\subsection{Material Synthesis}

The samples were prepared by mixing C-Ap and chitosan (1:1) and were added with petroleum white $(1: 1)$ to make them easy for application in the defect.

\subsection{Animal Model}

The use of animal protocol was approved by the Health and Medical Research Ethics Committee of The Medical Faculty, Universitas Gadjah Mada. The health status of the animals was monitored throughout the entire study. The animal experiment was performed according to the guidelines for animal experiments for scientific research of the Indonesian government. Twenty seven healthy adult male rats were used in this study because their good adaptatiton ability and resistance (The Human Society of The United States, 2006). Animal was housed in caged in the Integrated Research and Testing Laboratory (LPPT), Universitas Gadjah Mada. 


\subsection{Implantation}

Three treatment groups and one control group for each implantation time (1,2,3 weeks) were prepared: surgery was performed under general anesthesia that induced by an intramuscular injection of ketalar ketamin and xylasin. The animal's hind limbs was saved, washed and disinfected with povidone iodine. A longitudinal incision was made on the surface of both the left and right rat tibia. After exposure of the bone surface, a defect was created with $3 \times 1,25 \times 1$ $\mathrm{mm}^{3}$ with round and fissure dental drills using low rotational speeds and continuous cooling with saline solution. Sample was implanted into the defect and the soft tissue was closed in separate layers, using resorbable vicryl 2.0 sutures. Antibiotic and analgetic were administrated per oral as a safety procedure in preventing any bacterial infections.

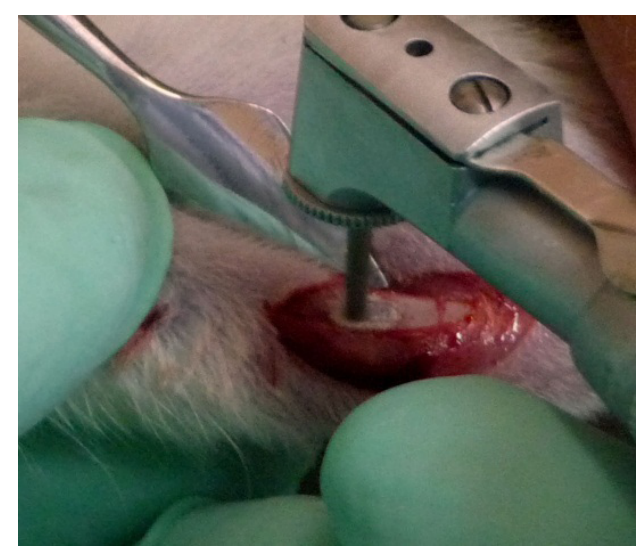

Fig. 1 Defect of rat tibia

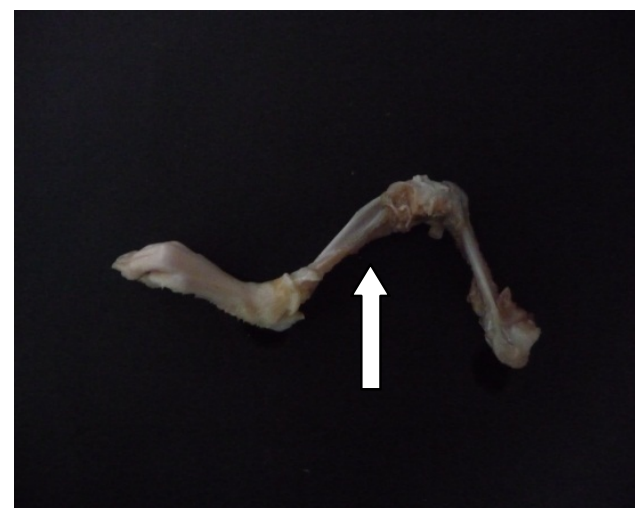

Fig. 2. Rat tibia

\subsection{Specimen Preparation}

After 1, 2 and 3 weeks, the rats were sacrificed and the bone were retrieved for evaluation. The tibias were excised and excessive tissue was removed. The bone was cut with a diamond saw and the retrieved area of defect were divided into smaller specimens, suitable for histological processing. Subsequently, the specimens were fixed in $10 \%$ formaldehyde for 24 hours. Plank and Rychlo method for decalcification and ethanol was used to dehydrated specimens. The sections were stained with Hematoxylin Eosin and examined by light microscopy.

\subsection{Statistical Analysis}

Data were statistically analyzed by two ways ANOVA continued by LSD test for a number of osteoblast and osteoclast. A $p$ values of $<0.05$ was considered statistically significant.

\section{Results}

\subsection{Macroscopic evaluation}

During the experimental period, all rats remained in good health. At the time of rat sacrifice, no inflammation or adverse tissue reaction were apparent.

\subsection{Histological evaluation}

Light microscopical analysis showed the bone ingrowth around the defect. Both treatment groups showed more newly bone than the control group in the same time implantation time. Two ways ANOVA analysis showed significant differences of osteoblast and osteoclast numbers between the groups $(p<0.05)$. The post hoc statistical analysis by LSD method showed significant difference only for the number of osteoblast between treatment and control $(p<0.05)$ but not for the number of osteoclast $(p<0.05)$. 


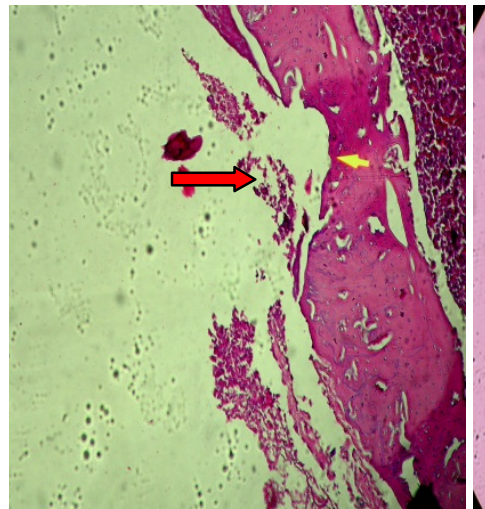

(a) C-Ap

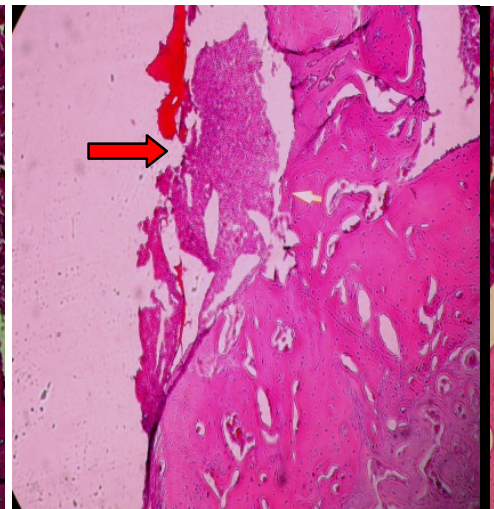

(b) C-Ap-chitosan

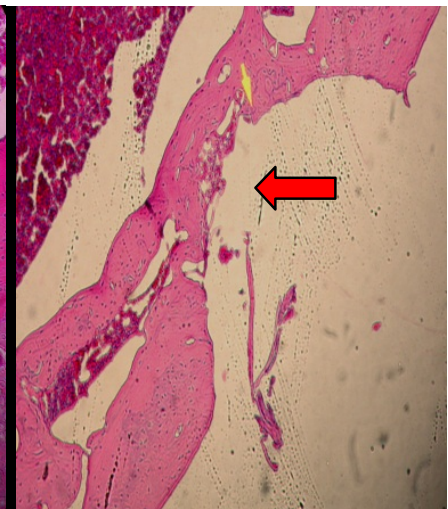

(c) Control

Fig.2 Light microscopy showed inflammatory cells around defect area in 1 week (100X)

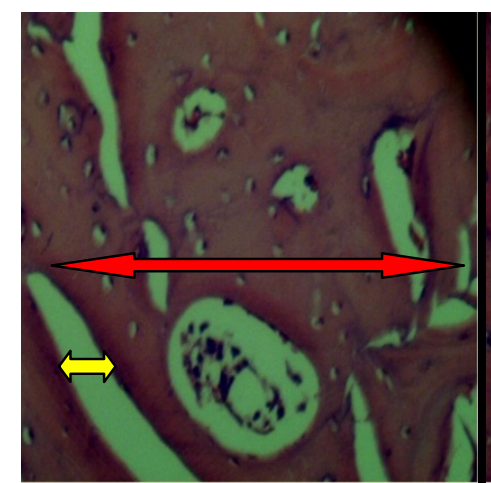

(a) C-Ap

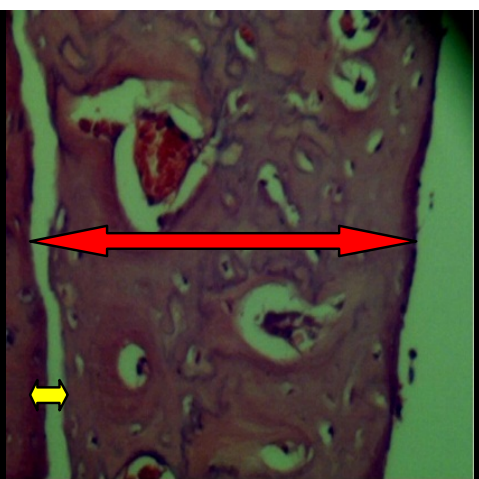

(b) C-Ap-chitosan

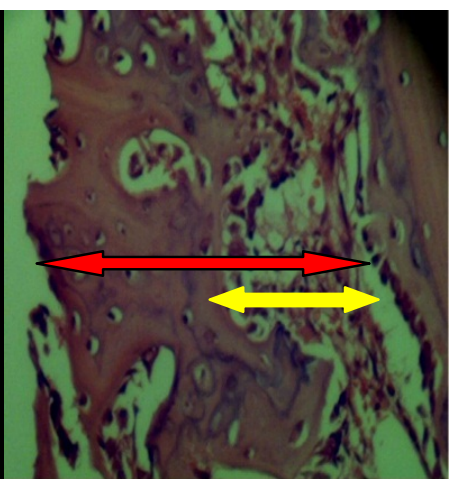

(c) Control

Fig. 3. Light microscopy in 2 weeks (350X). The red arrow showed the bone healing area.

There are a gap between the old and new bone (the yellow arrow)

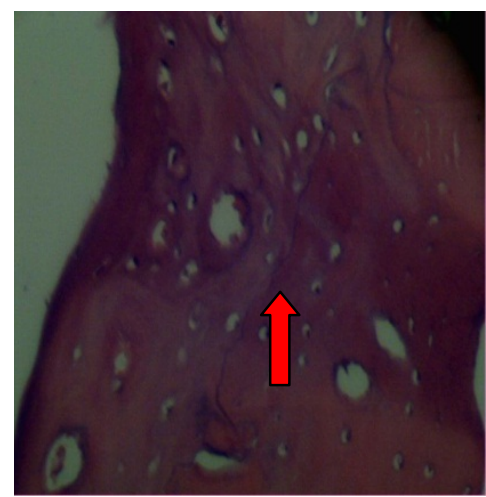

(a) C-Ap

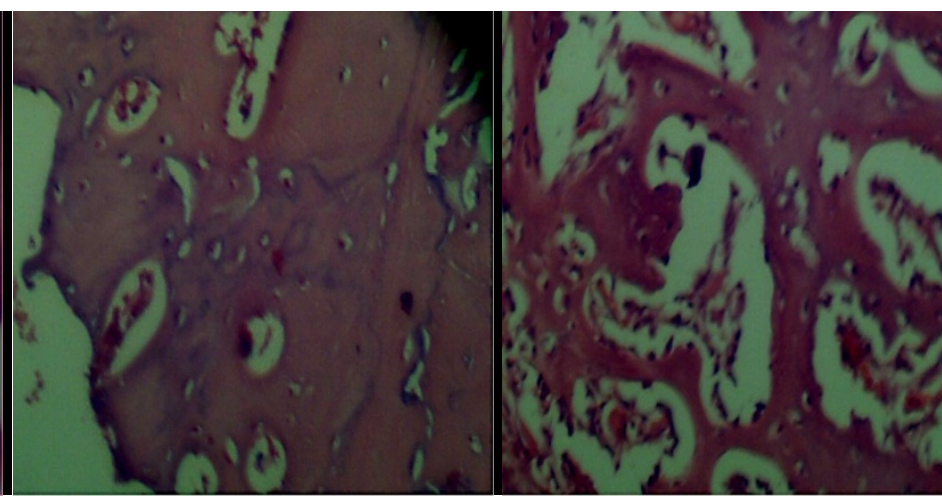

(b) C-Ap-chitosan (c) Control

Fig. 4 Light microscopy in 3 weeks (350X). Both (a) and (b) show more mature bone than (c) 


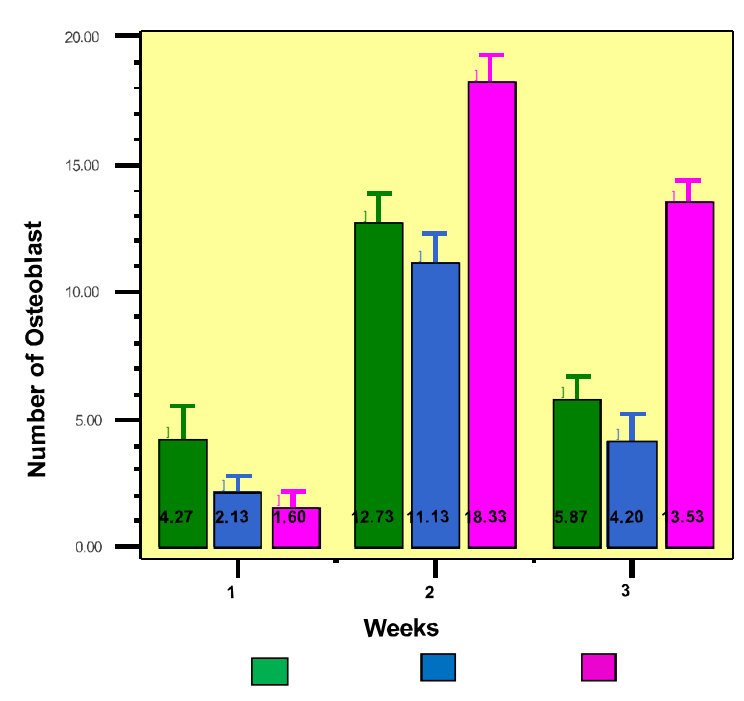

C-Ap C-Ap-Chi Control

Fig. 5 Histogram of osteoblast number in various implant time

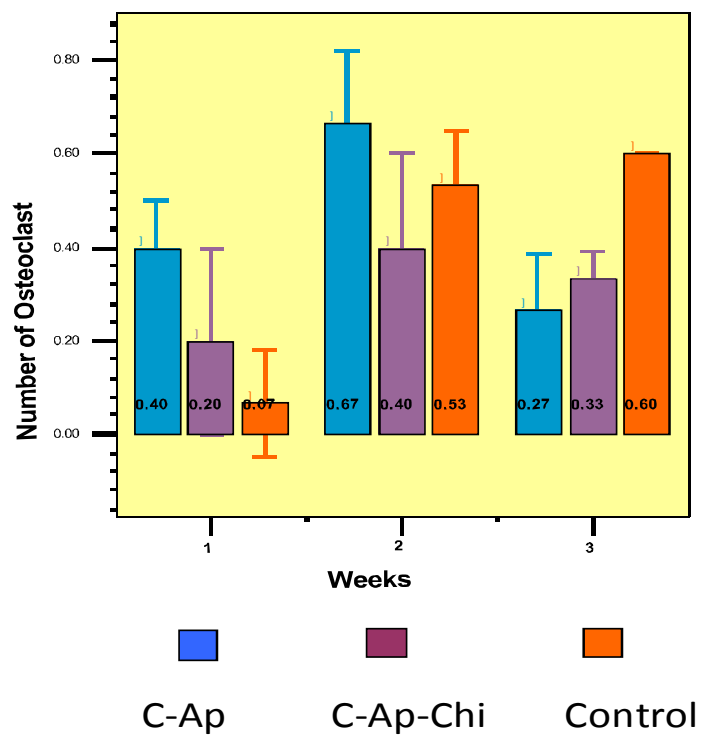

Fig. 6 Histogram of osteoclast number in various implant time

\section{Discussion}

The aim of this study was to evaluate the newly bone formation on tibia bone defect healing treated with C-Ap-chitosan bone substitute. This study compared number of osteoblast and osteoclast in a response to implantation of C-Ap and C-Ap-Chitosan on Sprague Dawley rats. The activity of osteoblast and osteoclast are potential biochemical marker of bone formation and which reveal the success of a bone graft to initiate, support and assist bone regeneration ${ }^{14}$. The results of this study confirmed that C-Ap and C-Ap-chitosan are potential to improve the bone regeneration. Meanwhile, C-Ap bone substitute showed faster bone healing process than C-Ap-chitosan.

Histological evaluation revealed that both the C-Ap and C-Ap-chitosan showed a higher number of osteoblast and osteoclast than the control group in 1 week implantation but it is opposite in 2 and 3 weeks. The number of osteblast indicated that bone formation process still happened. After the maturation process, the osteoblast will be calcified and become the osteocyt and continued to remodelling process by osteoclast. This result showed that the number of osteoblast in a control group was still higher than the treatment groups in 2 and 3 weeks implantation time. It indicated that the number of osteoblast in a treatment groups are faster to be calcified and remodelled by osteoclast. The decreasing number of osteoblast in 3 weeks implantation time indicated that the increasing calcified process of osteoblast to be an osteocyt.

Carbonate ions are closely related to human metabolism. Bicarbonates in blood and body fluids, whose concentration equilibrates with the carbonate content of bone apatites, contribute to the buffer mechanisms present ${ }^{14}$. This is an idea that osteoblast and osteoclast may adapt and respon to their surrounding environment, such as the carbonate concentration from bone substitute that is implanted in situ. Therefore, we believe that for a bone substitute biomaterials, C-Ap or C-Ap-chitosan are suitable and biocompatible and have ability to improve the bone healing.

Light microscopical analysis demonstrated that there were remaining chitosan in 1, 2 week. It is indicated that chitosan is resorbsed slower than C-Ap. The result showed that C-Ap alone was more effective to increase a number of osteoblast and osteoclast especially in 1 week implantation.

The result showed that a number of both osteoblast and osteoclast in a control group are highest in a 3 weeks. It is indicated that a bone still active to repair the defect while in the treatment group toward maturation process because almost of 
all the defect are covered by new bone (Fig.4) We can explain the reason of the result in a treatment groups that showed more osteocyt than osteoblast in 3 weeks. The treatment group was found to be more effective to stimulate bone regeneration than the control group.

Chitosan and its derivatives possess some special properties for use in regenerative medicine. A property of chitosan can be molded in various forms such as powder, paste, film, fiber and so forth for application with different demands. Chitosan can be combined with a variety of biomaterials such as alginate, hydroxyapatite, hyaluronic acid, calcium phosphate, polymethil metacrylate (PMMMA), poly-L- lactic acid (PLLA) and growth factors for potential application. Many chitosan derivatives are also biocompatible and non toxic with living tissues. Several injectable materials based on chitosan and its derivatives have been used as osteogenic bone substitutes ${ }^{15,16,17}$.

\section{Conclusion}

Based on the result, chitosan is a candidate to be used in tissue engineering as a bone substitute when it is combined with carbonate apatite. We suggest to modify the powder form to be a nanoparticle of chitosan to be easily resorbed in the body fluid.

\section{Acknowledgements}

Authors thank to faculty of dentistry, University of Gadjah Mada for the support to Anne H. Dewi to run this research and to UPT, BBPTK, LIPI Yogyakarta for the support of chitosan material.

\section{References}

1. Kalfas IH. 2001. Principles of bone healing. Neurosurg Focus, 10(4): Article 1:1-4.

2. Murugan R, Ramakrishna S. 2003. Bioresorbable composite bone paste using polysaccharide based nano hydroxyapatite. Biomaterials, 25: 3829-3835.

3. Doi Y, Iwanaga H, Shibutani T, Moriwaki Y, Iwayama Y. 1999. Osteoclastic responses to various calcium phosphates in cell culture. J. Biomed
Mater Res, 47: 424-433.

4. Mangano C, Scarano A, Vittoria P, Giovanna L, Adriano P. 2007. Maxillary sinus augmentation with a porous synthetic hydroxyapatite and bovine-derived hydroxyapatite: A comparative clinical and histologic study. J Oral Maxillofac Implants, 22: 980-986.

5. Wollf KD, Swaid S, Nolte D, Bockmann RA, Holzle F, Mai CM. 2004. Degradable injectable bone cement in maxillofacial surgery:indication and clinical experience in 27 patients. J CranioMaxillofac Surg, 32: 71-79

6. Browaeys H, Bouvry P, De Bruyn HA. 2007. Literature review on biomaterials in sinus augmentation procedures. Clin Implant Dent Relat Res, 9 (3): 166-77.

7. Hasegawa M, Doi Y, Uchida A. 2003. Cell-mediated bioresorption of sintered carbonate patite in rabbits. J Bone Joint Surg (Br), 85-B: 142-7.

8. Ivanova TI, Kamenetskaya F, Kol'tsov, AB, Ugolkov VL. 2001. Crystal structure of calcium-deficient carbonated hydroxyapatite. thermal decomposition. Journal of Solid State Chemistry, 160: 340-349.

9. Matsuya S, Udoh K, Nakagawa M, Ishikawa K. 2005. Preparation of carbonate apatite monolith by treatment of the set gypsum containing calcite in trisodium phosphate solution. Archieves of Bioceramics Research, 5: 256-260.

10. Ana ID, Mudjosemedi M, Sofro ASM, Leeuwenburgh SGC, Wolke JGJ, Ishikawa K, Jansen JA. 2007. Development of injectable carbonate apatite bone substitute based on phasetransformation of gypsum anf calcium hydroxyde: preliminart studies on factors influencing carbonate apatite synthesis. Trend, Technology, and Innovation in Comprehensive Oral Health Care. $29^{\text {th }}$ Asia Pasific Dental Conggres. Jakarta: 147.

11. Khan TA, Peh KK, Ch'ng HS. 2000. Mechanical, bioadhesive strength and biological evaluations of chitosan films for wound dressing. J Pharm Pharmaceut Sci, 3(3): 303-311.

12. Han S. 2005. Topical formulations of water soluble chitin as a wound healing assistant- evaluation on open wounds using a rabbit ear model. Fibers and Polymers, 6 (3): 219-223.

13. Yubao ZL, Aiping Y, Xuelin P, Xuejiang W, Xiang Z. 2005. Preparation and in vitro investigation of chitosan/ nano-hydroxyapatite composite used as bone substitute materials. Journal of Materials Sciences: Material in Medicine, 16:213-219.

14. Matsuura A, Kubo T, Doi K, Hayashi K, Morita K, Yokota R, Hayashi H, Hirata I, Okazaki M, Akagawa Y. 2009. Bone formation ability of carbonate 
apatite-collagen scaffold with different carbonate contents. Dental Material Journal, 28(2): 234-242

15. Chunmeng S, Ying Z, Xinze R, Meng W, Yongping S, Tianmin C. 2006. Therapeutic potential of chitosan and its derivatives in regenerative medicine. Journal of Surgical Research, 133: 185-192.

16. Hejazi R, Amiji M. 2003. Chitosan-based gastrointestinal delivery system. J Control Release, 89: 151.

17. Khor E, Lim LY. 2003. Implantable applications of chitin and chitosan, Biomaterials, 24: 2339. 